\title{
Atypical Retinoblastoma Presentation - A Challenge for the Treating Ophthalmologist
}

Siti Amra Abd Rahman ${ }^{1}$, Ahmad Salehuddin Mohammad², Safinaz Mohd Khialdin³ ${ }^{3}$ Siti Norzalehawati Sepain Jusoh ${ }^{4}$

\author{
${ }^{1,3}$ Department of Ophthalmology, University Kebangsaan Malaysia Medical Centre (UKMMC), Kuala \\ Lumpur, Malaysia. ${ }^{2}$ Department of Ophthalmology, University Malaya Medical Centre (UMMC), Kuala \\ Lumpur, Malaysia. ${ }^{4}$ Department of Ophthalmology, Hospital Sultanah Nurzahirah, Kuala Terengganu, \\ Terengganu Malaysia.
}

\section{INTRODUCTION}

Retinoblastoma is the most common primary intraocular malignancy in children. ${ }^{1}$ Incidence of retinoblastoma is constant worldwide at one case per 15000-20000 live births, which corresponds to about 9000 new cases every year.2,3 It usually occurs in children under five years of age and can lead to loss of vision, and in extreme cases, death. ${ }^{4}$

The common signs of its presentation are leukocoria, strabismus, secondary glaucoma, proptosis, anterior chamber inflammatory signs and spontaneous hyphaema. ${ }^{1}$ Prognosis for vision and survival has significantly improved due to timely diagnosis and improved treatment methods. ${ }^{5}$

\section{PRESENTATION OF CASE}

A 2.5-year-old girl was referred to the ophthalmology clinic for right eye white reflex associated with acute bilateral upper and lower limb weakness and urinary retention. Upon further history taking, right leukocoria was first noticed by her mother at 1.5 years and unfortunately, was only referred to an ophthalmologist at two years of age. Thorough investigations were done. The magnetic resonance imaging (MRI) radiographic findings were consistent with persistent foetal vasculature (Figure 1).

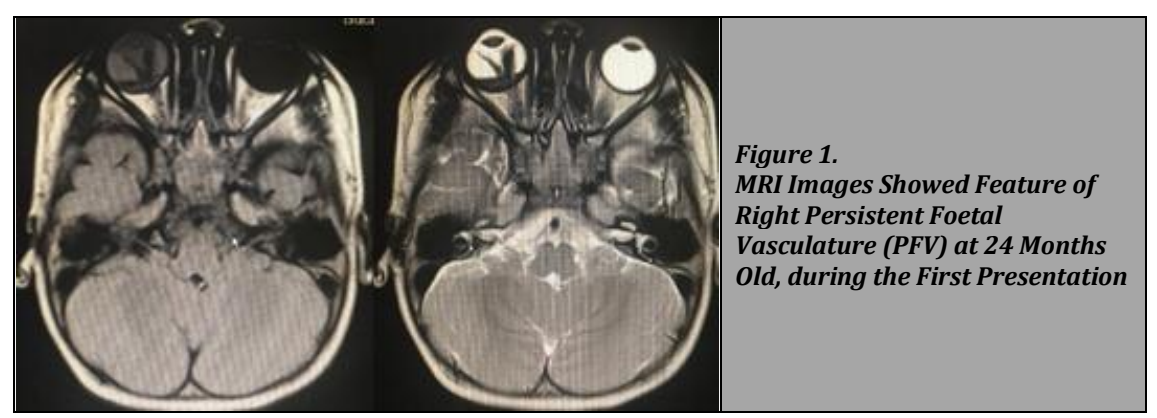

On this second presentation, ocular examination findings of the right eye revealed ciliary injection and generalized cornea haziness with early band keratopathy changes. The anterior chamber was deep with the presence of blood clots mixed with whitish materials on the iris stroma, which was highly suspicious of metastatic tissue. The right posterior segment was obscured by a retrolental reddish-yellowish hue. She was then proceeded with an ultrasonography study, in which there was no intrinsic mass calcification detected (Figure 2). Fortunately, her left eye revealed normal ocular findings. Clinically, it was strongly suspicious of right retinoblastoma.
Corresponding Author: Dr. Siti Amra Abd Rahman Department of Ophthalmology, University Kebangsaan Malaysia Medical Centre, Jalan Yaacob Latif, Cheras - 56000, Kuala Lumpur, Malaysia.

E-mail: sitiamra.abdrahman@gmail.com

DOI: $10.14260 /$ jemds/2021/720

How to Cite This Article:

Rahman SAA, Mohammad AS, Khialdin SM, et al. Atypical retinoblastoma presentation - a challenge for the treating ophthalmologist. J Evolution Med Dent Sci 2021;10(40):3554-3556, $10.14260 /$ jemds $/ 2021 / 720$

Submission 22-06-2021, Peer Review 17-09-2021, Acceptance 25-09-2021, Published 04-10-2021.

Copyright (C) 2021 Siti Amra Abd Rahman et al. This is an open access article distributed under Creative Commons Attribution License [Attribution 4.0 International (CC BY 4.0)] 


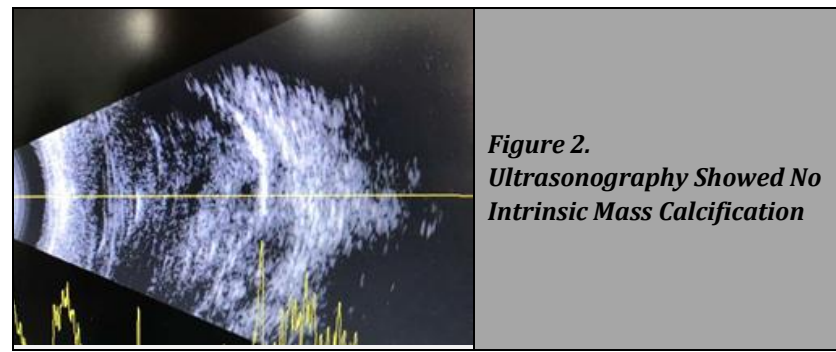

As expected, her repeated MRI study was highly suggestive of right retinoblastoma with spinal metastasis disease (Figure 3), which reported as right vitreous cavity mass with local and leptomeningeal extension metastases in the brain and spine.

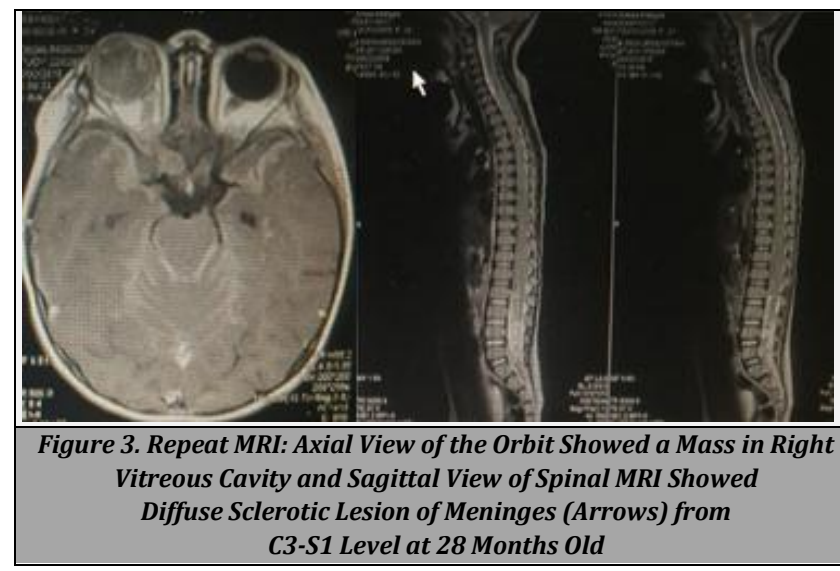

Alas, cerebrospinal fluid samples which were sent twice for full metastatic workup reported negative malignant cells. Nevertheless, she received three cycles of chemo reduction with intrathecal methotrexate and MRI orbit postchemotherapy showed significant reduction of the intraorbital lesion (Figure 4).

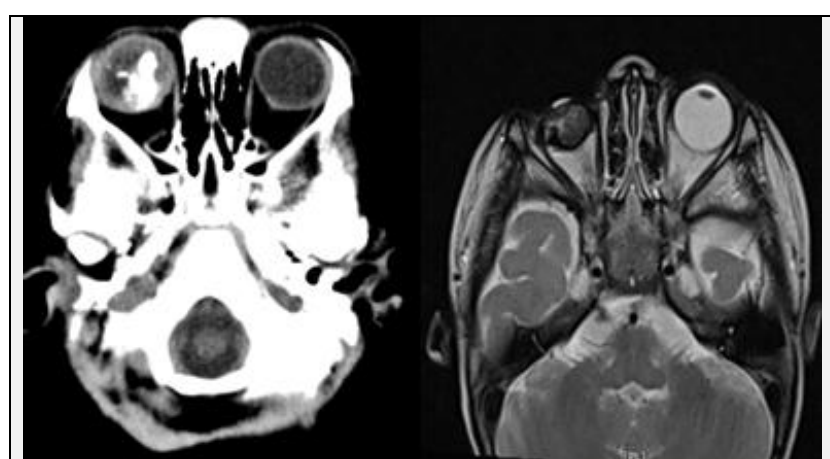

Figure 4. Computed Tomography (CT) Scan Pre-Chemo Reduction (Left) and MRI Post-Chemo Reduction (Right) Showed Radiological Evidence of Response to Treatment, during Which Cytological or Histological Evidence of Retinoblastoma Was Yet to be Obtained

The patient missed a few follow-up visits due to the enucleation being a hard decision for the parents. Her return for follow up was brought by new symptoms of right corneal ulcer with an anal abscess. Subsequently, right enucleation (Figure 5) with the implant was carried out after the third cycle of chemoreduction simultaneous with a permanent colostomy.

Histopathology revealed solitary retinoblastoma with Bruch membrane and focal choroidal invasion. There was no evidence of optic nerve invasion and the resected margin of the optic nerve was clear. She was planned for a total of nine cycles of chemotherapy based on spinal protocols. Unfortunately, she succumbed to death a few months later due to multi-organ failure.

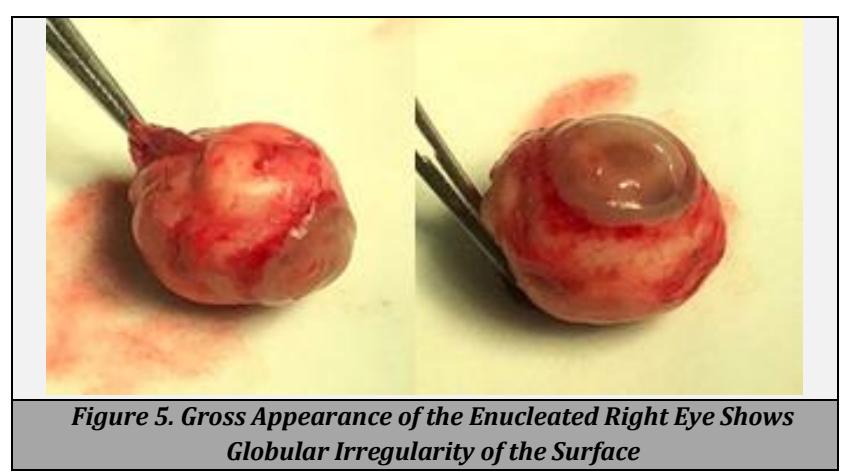

DISCUSSION

An almost similar atypical case of retinoblastoma was reported in 2014 where the authors described an 18-monthold child with a dystrophic hyaloid artery but unaccompanied by hyperplastic primary vitreous. The child also had other ocular features such as the cataractous lens and secondary glaucoma that lead to buphthalmos. Histopathology investigation of the enucleated eye confirmed the diagnosis of retinoblastoma. ${ }^{6}$

In our case, similarly, the persistent foetal vasculature (PFV) with secondary glaucoma was present. This posed a great challenge to our clinicians to make the right diagnosis until the histopathology results were obtained while other cytology results came back negative of any malignant cells.

An initial radiological finding of PFV had somewhat disapproved the working diagnosis of retinoblastoma and partly caused misdirection in the diagnosis which in turn, deviated subsequent actions by the clinicians.

Months later, only after the disease had progressed, the patient's re-presentation at our centre brought the suspicion of retinoblastoma but at a much higher level due to her new onset of neurological symptoms that were very suggestive of advancement to extraocular disease.

Again, our clinicians faced another diagnostic dilemma as they could not proceed with further evaluation. Both parents were adamant and refused any investigative procedure that included bone marrow biopsy and lumbar puncture.

They were also very hesitant for right eye enucleation which at this stage only aimed for diagnostic confirmation as the disease had already metastasized to the spine. This had further delayed the diagnostic process and treatment with chemotherapy.

Jamalia et al. also reported similar behaviour of the parents in 2010 where they lost $60 \%$ of patients at follow up due to refusal and inability to accept enucleation as a form of treatment. ${ }^{7}$ Some patients were lost in the middle of treatment because parents were unable to cope with the intensive treatment and frequency of the follow-ups. ${ }^{7}$ In most instances, the issues were more of parental motivations rather than financial constraints as treatments for this 
childhood cancer were highly subsidized by the government and National Cancer Council. ${ }^{7}$

The great association of leukocoria with the diagnosis of retinoblastoma should not be ignored by the clinician despite some other possible diagnosis of pseudo-retinoblastoma. Carol L. Shields et al. through their retrospective case series of 2775 cases referred for management of retinoblastoma found that there were only $22 \%$ (604 cases) of pseudoretinoblastoma. ${ }^{8}$ Persistent foetal vasculature (PFV) represented $28 \%$ of pseudo-retinoblastoma described in the study. ${ }^{8}$ Further narrowed to the 2-5 year old age group, only $7 \%$ were more likely to have confirmed diagnosis of PFV. ${ }^{8}$ Thus, while encountering such atypical cases, the treating clinician should have a high suspicion of retinoblastoma against all odds but at the same time be aware of possibilities of other various pseudo-retinoblastoma.

\section{CONCLUSION}

Atypical cases of retinoblastoma may lead to diagnostic dilemma as in this case; the initial radiographic MRI finding which was consistent with persistent foetal vasculature had caused misdirection and disapproved the early detection of retinoblastoma. The problem of late diagnosis and presentation must be tackled to improve prognosis and survival as well as the quality of life for the patients, especially in atypical cases. ${ }^{7}$

Financial or other competing interests: None.

Disclosure forms provided by the authors are available with the full text of this article at jemds.com.

\section{REFERENCES}

[1] Pandey AN. Retinoblastoma: an overview. Saudi J Ophthalmol 2014;28(4):310-5.

[2] Dimaras H, Kimani K, Dimba EA, et al. Retinoblastoma. Lancet 2012;379(9824):1436-46.

[3] Kivelä T. The epidemiological challenge of the most frequent eye cancer: retinoblastoma, an issue of birth and death. Br J Ophthalmol 2009;93(9):1129-31.

[4] McDaid C, Hartley S, Bagnall AM, et al. Systematic review of effectiveness of different treatments for childhood retinoblastoma. Health Technol Assess 2005;9(48):1145.

[5] Reddy SC, Anusya S. Clinical presentation of retinoblastoma in Malaysia: a review of 64 patients. Int J Ophthalmol 2010;3(1):64-8.

[6] Jakobiec FA, Rai R, Rashid A, et al. Dystrophic hyaloid artery remnants and other abnormalities in a buphthalmic eye with retinoblastoma. Surv Ophthalmol 2014;59(6):636-42.

[7] Jamalia R, Sunder R, Alagaratnam J, et al. Retinoblastoma registry report--hospital Kuala Lumpur experience. Med J Malaysia 2010;65(Suppl A):128-30.

[8] Shields CL, Schoenberg E, Kocher K, et al. Lesions simulating retinoblastoma (pseudoretinoblastoma) in 604 cases: results based on age at presentation. Ophthalmology 2013;120(2):311-6. 